\title{
KINETIC PARAMETERS OF THERMOLUMINESCENCE BASED ON ISOTHERMAL DECAY CURVES
}

\author{
Titas Paul, Antor Mahamudul Hashan \\ Ural Federal University, Yekaterinburg, Sverdlovsk Oblast, Russia
}

\begin{abstract}
The majority of the current techniques for getting the recurrence factors utilize the snare profundity making a few suppositions about the request for the energy. This causes irregularities in the announced benefits of catching boundaries due that the estimations of the actuation energy got by various techniques contrast obviously among them. At that point, it is important to utilize a technique autonomous of the snare profundity utilizing the isothermal iridescence rot (ILD) method. The catching boundaries related with the unmistakable shine pinnacle of $\mathrm{BeO}$ $\left(280^{\circ} \mathrm{C}\right)$ are accounted for using ILD strategy. As a check, the snare boundaries are additionally determined by sparkle bend shape strategy subsequent to disconnecting the conspicuous shine top by warm cleaning procedure. Our outcomes show an awesome arrangement between the catching boundaries determined by the two techniques. ILD strategy was utilized for deciding the catching boundaries of BeO. Results got applying this technique are in acceptable concurrence with those acquired utilizing different strategies, besides in the estimation of the recurrence factor.
\end{abstract}

Keyword - Isothermal decay curve, Thermoluminescence, Kinetic analysis, Glow curve, BeO.

\section{INTRODUCTION}

The development of new thermoluminescent (TL) materials for use in radiation dosimetry requires a deeper understanding of the capture parameters [1]. This can be achieved by analyzing the luminescence curve obtained after exposing the material to ionizing radiation in order to excite electrons from the valence band to the conduction band and back to metastable states in the bandgap above the Fermi level [2]. Then, when heated, the electrons can be released from the traps and recombine with the trapped holes that emit light.

There are many different methods to determine the trapping parameters [3] Most existing methods for obtaining frequency factors make use of the previous determination of the activation energy by making some assumptions about the order of the kinetics. This means that there are inconsistencies in the reported values of the trapping parameters due to significant differences in activation energy values obtained by different methods [4]. Then you need to use a method that does not depend on determining the depth of the trap. Isothermal luminescence decay (ILD) is an effective, reliable, and versatile method for estimating capture parameters because it has no preconditions and can be applied not only for first and secondorder kinetics but also for general order kinetics [5].

The characteristics of dosimeters used in the TL process are related to kinetic parameters, and these parameters quantitatively describe the centers of radiation collection. Detailed studies of kinetic parameters provide valuable information on the TL mechanism responsible for dosimetry applications [6]. A reliable study of TL material should be based on a good knowledge of the kinetic parameters.

This article reports on studies of $\mathrm{Be} 0$ in the field of kinetic parameters, which are important for the general description of the physical characteristics of a thermoluminescent material. As a check, the trap parameters are also calculated by glow curve shape(Chen's) method [7].

\section{Methodology}

Thermoluminescent (TL) response is recorded as a function of time while the sample is held at a constant temperature. The isothermal decay of luminescence is generally referred to as phosphorescence [8].

\section{A. Theory}

There are various methods that use the isothermal decay curve to determine the order of the kinetics, one using the change in the slope of the isothermal decay curve over time, and the other using the area under the isothermal decay plot [9]. However, the second presents two experimental problems. First, the criteria for choosing which temperature pair to use are not obvious, and the question of whether any pair can give similar results remains open. The second point is that this method requires that the total area under an isothermal decay curve be known, which in practical terms, calls for an extended measurement time. The dilemma here is that a prolonged measurement, while necessary and suitable to accurately an isolated peak, may cause simultaneous loss of signal from several peaks, particularly where such peaks are closely overlapping [10]. On the other hand, improperly approximating the total area would then inevitably introduce inaccuracies in the determination of $\mathrm{E}$. The main advantage of the first method is that it gives an estimation of the order of kinetics also, and is perhaps the only method un- 


\section{International Journal of Engineering Applied Sciences and Technology, 2021 \\ Vol. 6, Issue 1, ISSN No. 2455-2143, Pages 9-13 \\ Published Online May 2021 in IJEAST (http://www.ijeast.com)}

affected by temperature-dependent factors such as frequency factor and quantum efficiency. Then, we decide to use the first method. The TL power condition for the primary kinetics energy is:

$$
I=-\frac{d n}{d t}=n s e-\left(\frac{E}{K T}\right)
$$

Where I am the power at any instant, $\mathrm{n}$ is the quantity of populated traps at any instant, $\mathrm{E}(\mathrm{eV})$ is the snare depth, $s\left(s^{-1}\right)$ is the recurrence factor, $\mathrm{T}(\mathrm{K})$ is the temperature and $k(\mathrm{eV} \times$ $\left.K^{-1}\right)$ is the Boltzmann constant. The arrangement of force condition for the isothermal case:

$$
I(t)=n_{0} s e^{-\frac{E}{K T}} e^{-s t e^{-\frac{E}{K T}}}
$$

Where $n_{0}$ is the underlying grouping of the caught electrons. Therefore, for the main request case, plot of $\ln [I(t)] v s t$ should yield a straight line of slope $e^{-(E / K T)}$ :

$$
\ln I=-\left(s e^{-\frac{E}{K T}}\right) t+\left(\ln \left(n_{0} s\right)-\frac{E}{K T}\right)
$$

For general request energy the TL force condition is:

$$
I=-\frac{d n}{d t}=n_{0} s^{\prime} e^{-\frac{E}{K T}}
$$

Where $S^{\prime}=\left(s /\left(n_{0}{ }^{b-1}\right)\right)$ is the pre- exponential factor and $\mathrm{b}$ is the request for kinetics. The solution of intensity equation for the isothermal case:

$$
\left(\frac{I_{0}}{I}\right)^{\frac{b-1}{b}}-1=s e^{-\frac{E}{K T}}(b-1) t
$$

So for the overall request case, plot of $\left(I_{0} / I\right)^{((b-1) / b)}-1$ vs $t$ should yield a straight line where Io is the power at $t=0$ and $1<b \leq 2$. The experimental data are tried by picking by choosing different test values of $b$, among which the estimation of $b$ that gives the best fit as straight line compares to the request for energy. The slope of this straight-line is:

$$
(b-1) s e^{-\left(\frac{E}{K T}\right)}
$$

The determination of slope $(\mathrm{m})$ of straight line plots of $\left(I_{0} / I\right)^{-((b-1) / b)}-1$ vs $t$ at different temperatures will give the value of frequency factor from the following relation:

$$
\left(\frac{I_{0}}{I}\right)^{\frac{b-1}{b}}-1=m t+B
$$

The frequency factor value is obtained from the following equation:

$$
s=\frac{1}{(b-1)} \frac{m_{2}^{\frac{T_{2}}{T_{2}-T_{1}}}}{m_{1}^{\frac{T_{1}}{T_{2}-T_{1}}}}
$$

Where $m_{1}$ and $m_{2}$ are the slants comparing to the temperatures $T_{1}$ and $T_{2}$ respectively. Now to track down the warm actuation energy (E) a chart $\ln [m(t)]$ vs $1 / T$ ought to be plotted, which is a straight line. The slope $m=-E / K$ of this straight line graph will give the value of thermal activation energy $(E)$.

$$
\ln (m(t))=-\frac{E}{K T}+\ln (s(b-1))
$$

\section{B. Experimental Work}

The ILD strategy depends on recording the rot of the iridescence power with time at a consistent temperature [11]. In this strategy, three temperatures are chosen on the rising side of the gleaming top whose boundaries are to be resolved. After illumination of the example to the appropriate radiation portion, it is warmed to one of the chose temperatures and the rot of the glowing force is estimated, at a consistent temperature, as an element of time. This strategy is rehashed for two different temperatures.

At that point, trial information is tried to discover the request for kinetics. Disc molded $\mathrm{BeO}$ earthenware production, economically accessible in the un-doped structure under the business name Thermal ox 995 fabricated by Brush Beryllium Co., Elmore, $\mathrm{OH}$, the USA with complete contents of debasements not surpassing $0.5 \%$, with measurements: diameter $4 \mathrm{~mm}$ and thickness $1 \mathrm{~mm}$, were utilized in the TL measurements.

Before the assurance of motor boundaries, tests were submitted to warm strengthening treatment at $600^{\circ} \mathrm{C}$ during $30 \mathrm{~min}$ to eradicate any excess information. TL readings were made on a 


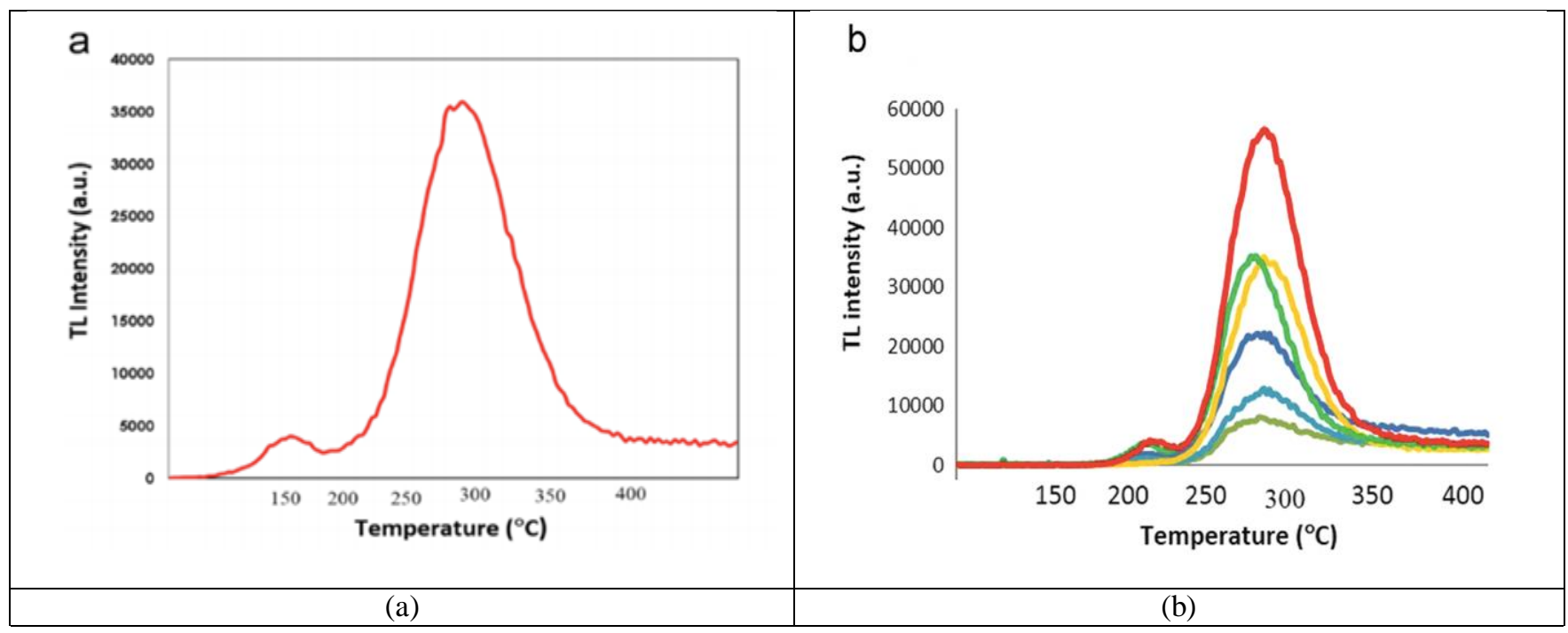

Figure 1. Thermoluminescent (TL) glow curves of $\mathrm{BeO}$ irradiated with $90_{s r} / 90_{Y}$ beta radiation. (a) $100 \mathrm{mGy}$;

(b) Different doses.

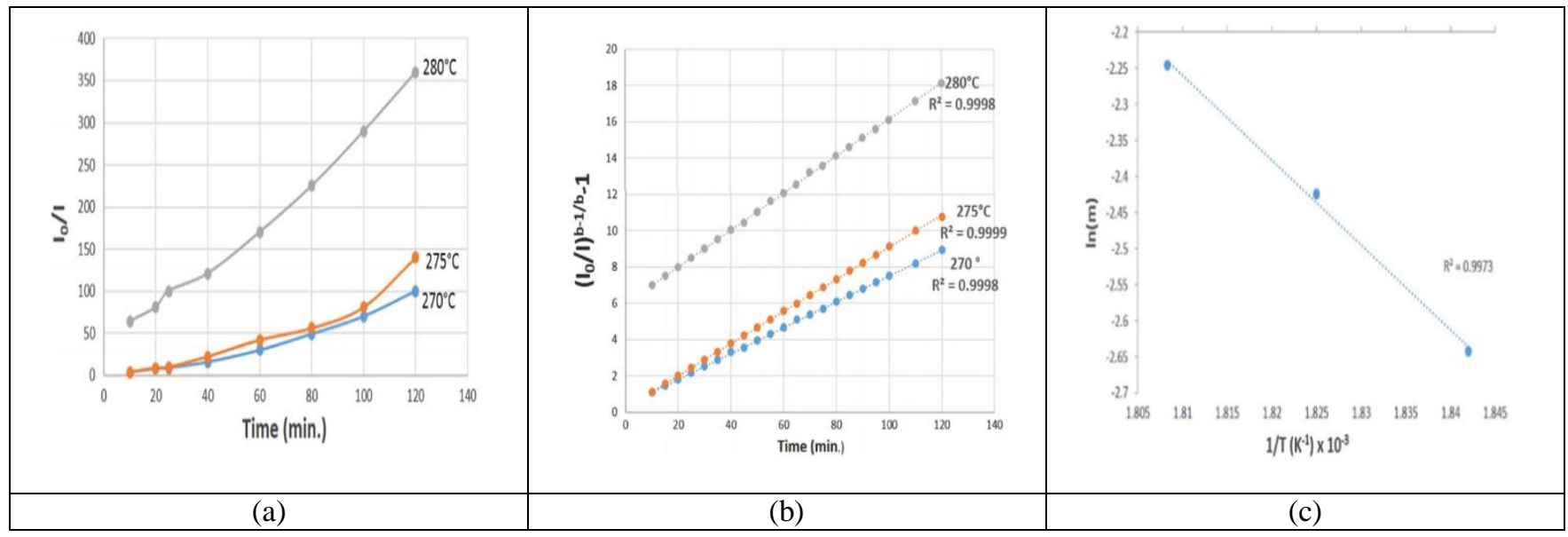

Figure 2. (a) Plot of $I_{0} / I$ vs time; (b) Plots of $\left(I_{0} / I\right)^{b-1 / b-1}$ vs time for temperatures of 270,275 and $280^{\circ} \mathrm{C}$;

(c) Plot of $\ln (m)$ vs $1 / T$.

Harshaw TLD Reader Model 3500 integrating the sign from 20 up to $400^{\circ} \mathrm{C}$ utilizing a warming rate of $5^{\circ} \mathrm{C} \mathrm{s}^{-1}$. All $\mathrm{TL}$ estimations were made in a nitrogen air to lessen the warm clamor coming about because of the warming planchet of the TL peruse. Dosimeters were illuminated at room temperature using $90_{s r} / 90_{Y}$ beta source $($ Emax $=2.28 \mathrm{MeV})$ at an assimilated portion of $100 \mathrm{mGy}$.

In request to compute the catching boundaries relating to $280^{\circ} \mathrm{C}$ shine top, three temperatures specifically 270,275 and $280^{\circ} \mathrm{C}$ were picked and rot of radiance force with time at these temperatures were recorded for tests lighted at an ingested portion of $100 \mathrm{mGy}$ for times from 10 to $120 \mathrm{~min}$. In first request energy, a plot of $\ln (\mathrm{I})$ against $t$ will bring about a straight line of incline $s e^{E / K T}$. In the instance of general request energy, a plot of $\left(I_{0} / I\right)^{((b-1) / b)-1}$ against $t$ ought to be a straight line. For this situation, the exploratory information is tried by picking diverse test estimations of, among which the estimation of $b$ that gives the best fit as straight line relates to the request for energy. On the off chance that the rot is observed at various temperatures, the incline is acquired. A plot of $\ln (m)$ against $1 / T$ gives a straight line of slope $E / k$ from which $E$ worth can be calculated. Frequency factor worth can be determined from Equation (8).

\section{RESUlts AND DisCUSSION}

Figure 1(a) displays the TL $\mathrm{BeO}$ luminescence curve obtained after exposure of the sample to beta radiation at an absorbed 


\section{International Journal of Engineering Applied Sciences and Technology, 2021 \\ Vol. 6, Issue 1, ISSN No. 2455-2143, Pages 9-13 \\ Published Online May 2021 in IJEAST (http://www.ijeast.com)}

dose of $100 \mathrm{mGy}$. The shape of the glow curve remains almost the same for all the exposures but the relative heights of the peaks change as a function of the absorbed dose, as is shown in Figure 1(b). The TL glow curve of $\mathrm{BeO}$ material recorded after irradiation with a $90_{s r} / 90_{Y}$ beta source showed two wellseparated peaks located at around 150 and $280^{\circ} \mathrm{C}$ at a heating rate of $5^{\circ} \mathrm{C} / \mathrm{s}$.

The plots of $I_{0} / I$ vs time were drawn at these temperatures which do not fit into the straight line nature, as shown in Figure. 2(a), which establish that the glow peak at $280^{\circ} \mathrm{C}$ does not obey first order kinetics. This indicates that TL mechanism of this peak is not associated with first order kinetics. Then plots of $\left(I_{0} / I\right)^{((b-1) / b)-1}$ vs time were drawn for different values of $\mathrm{b}$ $(1<b \leq 2)$. The best straight-line fitting was obtained for $\mathrm{b}=2$. This established that $280^{\circ} \mathrm{C}$ glow peak is associated with second order kinetics $(b=2)$. To find the thermal activation energy $(E)$, the slopes $(\mathrm{m})$ of these straight lines for the temperatures 270 , 275 and $280^{\circ} \mathrm{C}$ were noted and were plotted as $\ln (m)$ vs $1 / T$ as shown in Figure 2(b) which was a straight line as expected. The activation energy (E) was calculated from the slope $\left(\mathrm{m}^{\prime}=-\right.$ $\mathrm{E} / \mathrm{k}$ ) of this straight line and was found to be $1.01 \mathrm{eV}$.

The value of frequency factor (s) was found from the slopes of the straight lines of $\left(I_{0} / I\right)^{1 / 2-1}$ vs time plots at 270,275 and $280^{\circ} \mathrm{C}$ by using the Equation (8) and was found to be $1.8 \times$ $10^{9} \mathrm{~s}^{-1}$ (Figure 2(c)). Values of activation energy and frequency factor are shown in Table 1 comparing them with those obtained by Chen's method [12,13], where $\mathrm{E} \tau$, $\mathrm{E} \delta$ and $\mathrm{E} \omega$ are the values of the activation energy calculated by using the half-width in the raising part $(\tau)$, the half-width towards the descending part $(\delta)$ and the total half-width $(\omega)$ of the glow peak, respectively.

Table 1. TL parameters of $\mathrm{BeO}$ obtained by Chen's method.

\begin{tabular}{l|l|l}
\hline Method & $\boldsymbol{E}(\mathbf{e V})$ & $\boldsymbol{E}(\mathbf{e V})$ \\
\hline Isothermal decay & 1.01 & $1.8 \times 10^{9}$ \\
Chen $\tau$ & 1.14 & $9.3 \times 10^{10}$ \\
Chen $\delta$ & 1.07 & $2.3 \times 10^{11}$ \\
Chen $\omega$ & 1.12 & $2.2 \times 10^{11}$ \\
\hline
\end{tabular}

\section{CONCLUSION}

From the outcomes, we can reason that the estimation of actuation energy of $\mathrm{BeO}$ acquired applying the isothermal rot of TL is in acceptable concurrence with those got utilizing the shine bend shape Chen's altered strategy, yet the s esteem varies extensively. It is to be noticed that test mistakes alone are not answerable for such distinction, but rather the challenges in understanding of TL information are likewise reflected. Consequently, the vast majority of the strategies for ascertaining $s$ use trap profundity esteem $E$. Leaving to the side the trial blunders, regardless of whether one uses a similar information for acquiring the snare profundity, the qualities got by various techniques can vary considerably; henceforth the decision of an incentive for $\mathrm{E}$ in the resulting counts for $\mathrm{s}$ isn't unambiguous. As $\mathrm{E}$ enters as an example in the articulation for $\mathrm{s}$, a little variety in $\mathrm{E}$ prompts a significant change in determined estimations of $\mathrm{s}$.

Acknowledgments: We are thankful to "Ural Federal University, Yekaterinburg, Sverdlovsk Oblast, Russia" for their support and necessary facilities to carry out this study.

Conflicts of Interest: We declare that we have no financial or personal relationships with other people or organizations that can influence our work.

\section{REFERENCES}

[1] Azorín, J., Furetta, C., \& Scacco, A. (1993). Preparation and properties of thermoluminescent materials. Physica status solidi (A): Applied research, 138(1), 9-46.

[2] Moharil, S. V. (1984). A simple method for obtaining order of kinetics from isothermal decay of thermoluminescence. Journal of Physics D: Applied Physics, 17(11), 2301-2303.

[3] Azorín, J. (1986). Determination of thermoluminescence parameters from glow curves-I. A review. International Journal of Radiation Applications and Instrumentation Part D Nuclear Tracks and Radiation Measurements, 11(3), 159-166.

[4] Hashan, A. M., Haidari, A., Saha, S., \& Paul, T. (2021). Computer numerically controlled drawing robot based on computer-aided design. Journal of Mechanical, Civil and Industrial Engineering. doi:10.32996/jmcie.2021.2.1.2.

[5] Moharil, S. V. (1984). A simple method for obtaining order of kinetics from isothermal decay of thermoluminescence. Journal of Physics D: Applied Physics, 17(11), 2301-2303.

[6] Kitis, G., Furetta, C., Prokic, M., \& Prokic, V. (2000). Kinetic parameters of some tissue equivalent thermoluminescence materials. Journal of Physics D: Applied Physics, 33(11), 1252-1262.

[7] Chen, R. (1976). Methods for kinetic analysis of thermally stimulated processes. Journal of Materials Science, 11(8), 1521-1541.

[8] Manam, J., \& Sharma, S. K. (2005). Evaluation of trapping parameters of thermally stimulated luminescence glow curves in $\mathrm{Cu}$-doped Li2B4O7 phosphor. Radiation Physics and Chemistry (Oxford, England: 1993), 72(4), 423-427.

[9] Azorín, J., Gallegos, A., Rivera, T., Azorín, J. C., \& Khaidukov, N. (2007). Determination of the kinetic parameters of K2YF5: Tb from isothermal decay of thermoluminescence. Nuclear Instruments \& Methods in Physics Research. Section A, Accelerators, Spectrometers, Detectors and Associated Equipment, 580(1), 177-179.

[10] Azorin, J. (2014). Preparation methods of thermoluminescent materials for dosimetric applications: an overview. Applied Radiation and Isotopes: Including 
Data, Instrumentation and Methods for Use in Agriculture, Industry and Medicine, 83 Pt C, 187-191.

[11] Chithambo, M. L., \& Niyonzima, P. (2014). On isothermal heating as a method of separating closely collocated thermoluminescence peaks for kinetic analysis. Journal of Luminescence, 155, 70-78.

[12] Chen, R. (1977). On the analysis of thermally stimulated processes. Journal of Electrostatics, 3(1-3), 15-24.

[13] Chen, R. (1976). Methods for kinetic analysis of thermally stimulated processes. Journal of Materials Science, 11(8), $1521-1541$ 